\title{
Contribution à l'inventaire des bryophytes de corse: nouvelles données sur la présence de quelques espèces nouvelles, rares ou peu fréquentes
}

\author{
Achille Pioli
}

\begin{abstract}
PIOLI, A. (2015). Contribution to the inventory of Corsican bryophytes: new data on the presence of some new, rare or uncommon species. Candollea 70: 101-107. In French, English and French abstracts. DOI : http://dx.doi.org/10.15553/c2015v701a9

A bryological study on Corsican wetlands near the Col de Verde, and several surveys of the bryoflora in different regions conducted between 2008 and 2014, allowed to collect rare, uncommon and new species for the island. We report about Barbilophozia attenuate (Mart.) Loeske, Jamesoniella autumnalis (DC.) Steph. (hepatics), Hygroamblystegium tenax (Hedw.) Jenn. and Thamnobryum alopecurum var. gracillimum (Bot.) Dull (mosses), all new for the island. In addition, new localities are added for 5 hepatics and 20 mosses that are rare or uncommon in Corsica. Including these new discoveries, the island's bryoflora now comprises 3 hornworts, 156 liverworts and 419 mosses, for a total of 578 species.
\end{abstract}

\section{Résumé}

PIOLI, A. (2015). Contribution à l'inventaire des bryophytes de corse: nouvelles données sur la présence de quelques espèces nouvelles, rares ou peu fréquentes. Candollea 70: 101-107. En français, résumés anglais et français. DOI: http://dx.doi.org/10.15553/c2015v701a9

En Corse, une étude bryologique des zones humides près du col de Verde ainsi que des prospections de la bryoflore effectuées entre 2008 et 2014 dans différentes régions de l'île ont permis de récolter des espèces peu fréquentes, rares ou nouvelles pour l'île. Deux espèces d'hépatiques nouvelles pour la Corse (Barbilophozia attenuata (Mart.) Loeske, Jamesoniella autumnalis (DC.) Steph.) et deux taxons nouveaux de mousses (Hygroamblystegium tenax (Hedw.) Jenn., Thamnobryum alopecurum var. gracillimum (Bot.) Dull) sont signalés ainsi que de nouvelles stations pour 5 hépatiques et 20 mousses rares ou peu fréquentes dans l'île. Avec ces nouvelles découvertes, la bryoflore de l'île se compose dorénavant de 578 espèces comprenant 3 anthocérotes, 156 hépatiques et 419 mousses.

\section{Keywords}

Bryophytes - Corsica - Floristics

Adresse de l'auteur: Piedilacorte, 20212 Sant'Andrea di Bozio, Corse.

E-mail: pioliachille@yahoo.fr 


\section{Introduction}

Les bryophytes de Corse ont fait l'objet de nombreuses publications qui sont pour la plupart anciennes et datent des deux derniers siècles. Pour les plus récentes on notera le travail particulier de Bischler \& Jovet-Ast (1973) sur les hépatiques et, entre 1974 et 2005, une vingtaine de publications de J.-P. Hébrard (voir liste in Sotiaux et al., 2007) sur la végétation muscinale des forêts et maquis de l'île ainsi que sur des espèces rares. En 2007 la check-list des bryophytes de Corse (Sotiaux et al., 2007) a fait un état récapitulatif des bryophytes de l'île, présentes ou répertoriées dans la bibliographie, complété l'année suivante par un additif (Sotiaux et al., 2008). Ces deux publications font état de 95 espèces nouvelles pour la Corse (72 mousses et 23 hépatiques). Il ressort de ce travail de synthèse, que l'île présente aussi 24 espèces répertoriées dans la liste rouge UICN (EECB, 1995), ainsi que la mousse Buxbaumia viridis (DC.) Moug. \& Nestl. relevant de la directive européenne «Habitats». Une seule mousse endémique, Leptodon corsicus Enroth, A. Sotiaux, D. Quandt \& Vanderp. (Sotiaux et al., 2009) a été découverte à ce jour dans l'île. Avec ces connaissances, la bryoflore de l'île se composait jusque-là de 575 espèces comprenant 3 anthocérotes, 154 hépatiques et 418 mousses.

Toutes ces études ont montré que la Corse présente une diversité bryologique importante, parmi les îles de la zone ouest-méditerranéenne et atlantico-méditerranéenne (Sotiaux et al., 2007). Cette richesse est due notamment aux particularités de son relief qui permet l'installation de multiples microclimats et formations végétales vasculaires. Cependant certaines espèces méditerranéennes sont absentes de l'île soit par manque de connexion terrestre ou difficulté d'immigration due à la distance jouant un rôle de filtre, soit par absence de stations favorables.

Les espèces holarctiques, cosmopolites et acidophiles dominent sur l'ensemble de la bryoflore. Dans l'étage subalpin, on note une végétation muscinale pauvre en espèces, avec un recouvrement faible, à cause des conditions climatiques difficiles, due à une période estivale trop sèche (HÉBRARD, 1978). Un certain nombre de muscinées, répandues aux étages alpin et subalpin des Alpes franco-italiennes descendent en Corse dans les vallées encaissées où elles trouvent des conditions plus favorables. Pour les hépatiques la montagne corse offre également une faible représentation des éléments artico-alpins dont les Alpes et les Pyrénées sont riches (Geissler, 1981). Mais elle est aussi insuffisamment explorée. Enfin la bryoflore de l'île présente un niveau d'endémisme très éloigné de ce que l'on peut observer chez les plantes vasculaires. Une seule espèce endémique est répertoriée à ce jour, alors que ces dernières sont bien représentées (11\%) parmi les plantes vasculaires. Cela n'est toutefois pas nécessairement surprenant, les populations de bien des bryophytes étant génétiquement interconnectées sur de longue distance par le vent (Sotiaux et al., 2007).

\section{Méthodologie}

Les résultats présentés ici sont le fruit de deux études distinctes. La première est une étude réalisée à la demande du Conservatoire Botanique National de Corse (CBNC) dans le but de répertorier les zones humides situées aux alentours du Col de Verde (Corse du Sud) et d'en effectuer un inventaire au niveau des bryophytes. Dans ce cadre, neuf zones humides ont été étudiées et six d'entre-elles, situées entre $1270 \mathrm{~m}$ et $1470 \mathrm{~m}$, renfermaient des espèces rares ou nouvelles pour la Corse. Certaines recelaient également des espèces protégées localement comme Sphagnum auriculatum Schimp., S. palustre L., S. subnitens Russow \& Warnst. et $S$. subsecundum Nees. La seconde étude est une prospection beaucoup plus large entre 2008 et 2014 dans différentes régions de Corse où nous avons récolté ou parfois simplement noté les espèces rares, peu fréquentes ou nouvelles pour la flore de l'île.

Sont considérées rares les espèces citées de moins de 10 localités, en se référant à la Check-list des bryophytes de Corse (Sotiaux et al., 2007).

La nomenclature utilisée est celle de Grolle \& LoNG (2000) pour les hépatiques et celle de HiLl et al. (2006) pour les mousses. Le milieu dans lequel elles ont été inventoriées est indiqué en se référant aux associations végétales en place (Gamisans, 1999). Les espèces apparaissent, par ordre alphabétique, en deux chapitres: hépatiques et mousses. Les sigles $2 \mathrm{~A}$ et $2 \mathrm{~B}$ indiquent les deux départements de la Corse (Corse du Sud et Haute Corse). Un rappel des connaissances actuelles de leur chorologie dans l'île est donné.

\section{Résultats}

Les espèces nouvelles, rares ou peu fréquentes dans l'île sont signalées ci-dessous par ordre alphabétique des hépatiques puis des mousses.

\section{Hépatiques}

\section{Aneura maxima (Schiffn.) Steph.}

- 2A, Forêt territoriale de Marmanu, en bordure du chemin de randonnée GR20, cortège floristique vasculaire ayant des affinités avec le groupement de marais à laiches Caricetum intricatae caricetosum, mais aussi avec la mégaphorbiaie du Valeriano rotundifoliae-Adenostyletum briquetii [NM133528], $1390 \mathrm{~m}$, 3.10.2013, Pioli, A. s.n. (Hb. CBNC).

Espèce rare, signalée une seule fois, en forêt de Ghisoni (Sotiaux et al., 2007).

Barbilophozia attenuata (Mart.) Loeske

- 2B, Forêt de Valdoniellu, vers la voie d'accès au lac de Lavigliolu [UTM: NM 964796], exposition nord, fissure dans paroi rocheuse, en touffe avec la mousse Racomitrium elongatum, zone rocheuse granitique, dalles, parois verticales, avec alternance de fruticée 
naine à genêts de Lobel, bouquet d'aulnes odorants et pelouses rases, $1800 \mathrm{~m}$, sol acide, 29.7.2011, Pioli, $A$. (Hb. privé 20110729013).

Espèce nouvelle pour la Corse. Elle est présente en France et presque partout sur le continent européen. (SCHUMACKer \& VANA, 2005; Ros et al., 2007).

\section{Calypogeia azurea Stotler \& Crotz}

- 2A, Forêt territoriale de Marmanu, en bordure du chemin de randonnée GR20, cortège floristique vasculaire ayant des affinités avec le groupement de marais à laiches Caricetum intricatae caricetosum, mais aussi avec la mégaphorbiaie du Valeriano rotundifoliae-Adenostyletum briquetii [NM133528], $1390 \mathrm{~m}$, 3.10.2013, Pioli, A. s.n. (Hb. CBNC).

Espèce rare, signalée au lac Melo (Geissler, 1981).

Jamesoniella autumnalis (DC.) Steph.

- 2A, Forêt territoriale de Marmanu, en bordure du chemin de randonnée GR20, sur tronc de pin laricio au sol, en état de pourrissement avancé, et très humide, cortège floristique vasculaire ayant des affinités avec le groupement de marais à laiches Caricetum intricatae caricetosum, mais aussi avec la mégaphorbiaie du Valeriano rotundifoliae-Adenostyletum briquetii, [NM133528], $1390 \mathrm{~m}$, 3.10.2013, Pioli, A. s.n. (Hb. CBNC).

Espèce nouvelle pour la Corse. Espèce subboréale montagnarde largement répandue dans l'hémisphère nord (DüLL, 1983).

Lejeunea lamacerina (Steph.) Schiffn.

- 2A, Forêt territoriale de Marmanu, en bordure du chemin de randonnée GR20, zone humide provenant des divagations d'un petit torrent, cortège floristique vasculaire relèvant du Valeriano-Adenostyletum briquetii, dans une hêtraie [NM133528], $1430 \mathrm{~m}$, 3.10.2013, Pioli, A. s.n. (Hb. CBNC). 2005).

Espèce rare, signalée à Sisco dans le cap Corse (SkrzypczaK,

Scapania irrigua (Nees) Nees

- 2A, Forêt territoriale de Marmanu, en bordure du chemin de randonnée GR20, cortège floristique vasculaire ayant des affinités avec le groupement de marais à laiches Caricetum intricatae caricetosum [NM154529], 1280 m, 3.10.2013, Pioli, A. s.n. (Hb. CBNC); mais aussi avec la mégaphorbiaie du Valeriano rotundifoliae-Adenostyletum briquetii [NM133528], 1390 m, 3.10.2013, Pioli, A. s.n. (Hb. CBNC).

Espèce rare, signalée à Calacuccia et à Capelluccia dans le massif de Cagna (Camus, 1902; Geissler, 1981).
Trichocolea tomentella (Ehrh.) Dumort.

- 2A, Forêt communale de Palneca [UTM: NM 700897], peuplements de pin laricio avec sous étage de hêtre, station située dans une zone humide occupant une superficie d'environ $2500 \mathrm{~m}^{2}$, avec quelques aulnes glutineux et saules et à végétation vasculaire dont l'association s'apparente à l'Athyrio-Gentianetum, $1170 \mathrm{~m}$, exposition ouest, substrat granitique, 16.10.2008, Pioli, A. (Hb. privé 20081016002).

Nouvelle et troisième station répertoriée pour cette espèce très rare: le cortège des principales espèces de bryophytes est le suivant: Aneura pinguis, Conocephalum conicum, Ctenidium molluscum, Hookeria lucens, Palustriella commutata, Plagiochila porelloides, Plagiomnium undulatum, Pedinophyllum interruptum, Pellia endiviifolia, Riccardia chamedryfolia, Rhizomnium punctatum. On notera sur cette station la présence d'espèces de milieux riches en bases, peu communes sur granites, comme: Ctenidium molluscum, Palustriella commutata ou encore parmi les plantes vasculaires Geranium nodosum. Il est également à noter, sur le site, la présence d'un touradon remarquable de la mousse Hookeria lucens, d'environ $1 \mathrm{~m}^{2}$.

\section{Mousses}

Andreaea frigida Huebener

- 2B, vallée de la Restonica au déversoir du lac de Melu [UTM: NM019735], sur rochers siliceux avec aulnaie odorante aux alentours, à végétation vasculaire relevant de l'Alnetum suaveolentis alnetosum, $1711 \mathrm{~m}$, 6.10.2011, Pioli, A. (Hb. privé 16/2014).

Espèce rare en Corse, mentionnée autrefois dans la région du Monte Cinto (Parriat, 1951; Sotiaux et al., 2007). Elle figure sur la Liste Rouge de l'UICN (EECB, 1995).

\section{Antitrichia californica Sull.}

- 2A, Forêt de San Antone, aux alentours du Col de Verde, sur rocher le long du GR20 [UTM: NM150528], dans un peuplement forestier composé par un mélange de pin laricio et de hêtre avec quelques sapins pectinés, à végétation vasculaire dont l'association relève du Poo-Fagetum sylvaticae fagetosum, 1340 m, 7.7.2012, Pioli, A. (Hb. privé 20120707008).

Espèce rare en Corse, signalée dans la région d'Ajaccio à Punta Pozzo di Borgo, dans la région de Vivario au col de Morello (Hébrard, 1986) ainsi que dans le San Pedrone (Sotiaux et al., 2007).

\section{Breutelia chrysocoma (Hedw.) Lindb.}

- 2A,Forêt communale de Corrano,peuplementcomposé d'un taillis sous futaie de chêne vert avec maquis à bruyère et arbousier, dans le vallon de Morodesi (thalweg), dans une zone claire de maquis bas composé par 
Erica arborea, Phyllerea latifolia, Quercus ilex, Smilax aspera, Rubia peregrina, dont l'association relève de l'Erico-Arbutetum phillyreetosum latifoliae, exposition nord-ouest, substrat granitique, sol acide, aucun pied avec sporophyte, $400 \mathrm{~m}, 2.3 .2011$, Pioli, $A$. (Hb. privé 20110302002); 2A. Forêt communale de Corrano, le long de la route départementale D757, sur le talus rocheux, granitique, à végétation vasculaire composée d'une futaie de chêne vert avec maquis, dont l'association s'apparente à l'Erico-Arbutetum phillyreetosum latifoliae, aucun pied avec sporophyte, 7.3.2011, Pioli, A. (Hb. privé 20110307016).

Espèce peu fréquente en Corse, connue de la région d'Ajaccio, de Cauro, Bastia (Teghime), et retrouvée dans la région de Porto et dans les Calanche de Piana (HéBrard, 1986). Elle occupe de grandes surfaces sur les stations répertoriées.

Buxbaumia viridis (DC.) Moug. \& Nestl.

- 2B, Forêt communale d'Asco, peuplements composés de pin laricio s'apparentant au Galio - Pinetum laricii anthyllidetosum hermanniae, station située dans la zone humide de Valentinu [NM948951], à association se rapprochant de l'Athyrio-Gentianetum gentianetosum, en exposition nord, $1160 \mathrm{~m}$, quatre pieds sur un talus suintant tapissé par les restes très décomposés d'un tronc de pin laricio au sol, pas de bryophytes compagnes à proximité, 28.7.2010, Pioli, A. observation; $2 \mathrm{~B}$, Forêt communale d'Albertacce, canton de Calasima [NM922976], forêt de pin laricio à exposition nord-est, $1310 \mathrm{~m}$, sept pieds sur un tronc de pin laricio au sol, pas de bryophytes compagnes à proximité, végétation vasculaire s'apparentant au Galio-Pinetum laricii anthyllidetosum hermanniae, 29.6.2011, Pioli, A observation; 2B, Forêt communale de Vivario, vallée du Manganello, au lieu dit Grottacia [NM072677], forêt mélangée de hêtre et de pin laricio, exposition nord, $1150 \mathrm{~m}$, quatre pieds sur une souche de pin laricio, pas de bryophytes compagnes à proximité, seuls quelques thalles primaires de Cladonia, végétation vasculaire s'apparentant au Poo-Fagetum sylvaticae fagetosum, 1.6.2011, Pioli, A. observation; 2B, Forêt communale de Vivario, station proche de la précédente dans la Vallée du Manganello, sur le GR 20 [NM073677], peuplements mélangés de hêtre et de pin laricio d'altitude, exposition nord, $1100 \mathrm{~m}$, treize pieds sur une souche de pin laricio, colonisée par les mousses Hypnum cupressiforme, Dicranum tauricum et le lichen Cladonia coniocraea, végétation vasculaire s'apparentant au Poo-Fagetum sylvaticae fagetosum, 1.6.2011, Pioli, $A$. observation.
Espèce protégée (annexe 2 de la directive européenne «Habitats»). Peu fréquente, connue des hêtraies-sapinières et des forêts de pin laricio, dans les forêts d'Aitone, Valdoniellu, Rospa Sorba, Noceta, Restonica, Vizzavona, Marmano, San Antone et Palneca (HÉBrard, 2005 ; Pioli, 2006). Les nouvelles stations ont été découvertes dans des forêts où nous suspections sa présence.

Campylium stellatum (Hedw.) C. E. O. Jensen

- 2A, Forêt territoriale de Sant'Antone, tourbière de pente à végétation vasculaire dont l'association relève du Caricetum intricatae caricetosum, située dans une hêtraie-sapinière avec des pins laricio dominants [NM156529], 1290 m, 13.9.2013, Pioli, A. s.n. (Hb. CBNC); 2A, Forêt territoriale de Sant'Antone, tourbière de pente à végétation vasculaire dont l'association relève du Caricetum intricatae caricetosum, située dans une hêtraie avec des pins laricio, aulnes glutineux et aulnes odorants [NM15 4529], $1280 \mathrm{~m}$, 16.9.2013, Pioli, A. s.n. (Hb. CBNC).

Espèce rare, signalée dans la forêt d'Aïtone, dans la vallée du Tavignano près de Corte et au lac de Nino (CAmus, 1902, 1903; SarRasat, 1931).

Campylophyllum calcareum (A. C. Crundwell \& Nyholm) Hedenäs

- 2A, Forêt territoriale de Marmanu, en bordure du chemin de randonnée GR20, tourbière de pente, avec une configuration de pozzines, à cortège floristique de plantes vasculaires se rattachant au PinguiculoTrichophoretum cespitosi narthecietosum, boisée par bouquets, sapins, pins laricio, hêtres, avec une présence importante des aulnes odorants, à couvert de 4/10 [NM132528], 1457 m, 3.10.2013, Pioli, A. s.n. (Hb. CBNC).

Espèce rare, signalée au col d'Arcarota (Sotiaux et al., 2008).

Campylopus introflexus (Hedw.) Brid.

- 2A, versants de la Montagne de Cagna, en forêt communale de Pianotoli Caldarello/Zerubia, dans la sapinière dont l'association est à rapprocher du Poo-Fagetum sylvaticae abietietosum, 18.6.2008, Pioli, $A$. (Hb. privé 20080618002); 2B, forêt communale de Moltifao, tourbière de Valdo [UTM: NM128031], sur des touradons d'Osmunda regalis, dans une association s'apparentant à l'Hyperico-Alnetum osmundetosum, pas de bryophytes compagnes, 4.6.2010, Pioli, A. (Hb. privé 20100604001).

Espèce invasive, signalée jusqu'ici en forêt territoriale de Melaghia (Sotiaux et al., 2008). 
Cratoneuron filicinum (Hedw.) Spruce

- 2A, Forêt territoriale de Marmanu, en bordure du chemin de randonnée GR20, zone humide provenant des divagations d'un petit torrent, cortège floristique vasculaire relèvant du Valeriano-Adenostyletum briquetii, dans une hêtraie [NM133528], $1430 \mathrm{~m}$, 3.10.2013, Pioli, A. s.n. (Hb. CBNC); 2A, Forêt territoriale de Marmanu, en bordure du chemin de randonnée GR20, cortège floristique vasculaire ayant des affinités avec le groupement de marais à laiches Caricetum intricatae caricetosum, mais aussi avec la mégaphorbiaie du Valeriano rotundifoliae-Adenostyletum briquetii [NM133528], 1390 m, 3.10.2013, Pioli, A. s.n. (Hb. CBNC).

Espèce rare, signalée à Tenda (CAmus, 1895).

Homalothecium lutescens (Hedw.) H. Rob.

- 2B, Sant'Andrea di Bozio, talus le long de la route départementale qui mène de Piedilacorte au col de Campodonico [NM253823], $890 \mathrm{~m}$, touffe sur racines, végétation vasculaire dont l'association relève du $\mathrm{Poo-}$ Fagetum sylvaticae, 15.12.2011, Pioli, A. (Hb. privé 20111215001); 2B. Sant'Andrea di Bozio, Arbitro [NM243827], $750 \mathrm{~m}$, zone à serpentinites (Rajina) en bordure de la route départementale, touffe sur talus terreux, avec Genista corsica, Cardamine plumieri, Genista salzmannii, Scrophularia canina, ... à végétation vasculaire se rattachant plutôt à l'association du Stachydi-Genistetum corsicae, 29.4.2013, Pioli, A. (Hb. privé 2013/042923).

Espèce rare en Corse, signalée dans la région de Rusio et du San Petrone (Bocca al Prato, Campodonico; Sotiaux et al., 2008).

Homalothecium philippeanum (Spruce) Schimp.

- 2B, Forêt de San Petru d'Accia; sur le chemin d'accès au sommet du San Petrone, versant ouest [NM 258945], dans la hêtraie, sur rocher, à végétation vasculaire du Poo-Fagetum sylvaticae, 10.7.2011, Pioli, A. (Hb. privé 20110701003); 2B, Forêt communale de Sant'Andrea di Bozio [NM254820], 1020 m, hêtraie, talus le long de la piste forestière, dans une touffe de Neckera complanata sur racine de hêtre, végétation vasculaire dont l'association relève du Poo-Fagetum sylvaticae, 9.12.2012, Pioli, A. (Hb. privé 20121209005); 2A, Forêt territoriale de Marmanu, en bordure du chemin de randonnée GR20, zone humide provenant des divagations d'un petit torrent, cortège floristique vasculaire relevant du Valeriano-Adenostyletum briquetii, dans une hêtraie [NM133528], 1430 m, 3.10.2013, Pioli, A. s.n. (Hb. CBNC).
Espèce rare en Corse, signalée sur le San Petrone (Sotiaux et al., 2007, 2008).

Hygroamblystegium tenax (Hedw.) Jenn.

- 2B, Forêt de Valdoniellu, aux alentours des bergeries de Capanelle, vallon du Lavigliolu [NM965795],1580 m, sur rochers granitiques, végétation vasculaire composée d'une fruticée naine à genêts de Lobel, s'apparentant au Paronichio-Armerietum genistetosum, avec quelques vieux pins laricio isolés, sur sol acide, 29.7.2011, Pioli, $A$. (Hb. privé 20110729017); 2B, Forêt de Valdoniellu, vallon du Lavigliolu [NM964796], 1780 m, accès au lac de Lavigliolu, zone rocheuse granitique, dalles et parois verticales, végétation vasculaire éparse composée de fruticées naines à genêts de Lobel, sur sol acide, 29.7.2011, Pioli, A. (Hb. privé 20110729020).

Espèce nouvelle pour la Corse. Elle est répandue sur le pourtour méditerranéen (Ros et al., 2013).

Hygrohypnum luridum (Hedw.) Jenn.

- 2A, Forêt territoriale d'Aitone [MM872796],1200 m, dans le lit d'un ruisselet, sur rocher, peuplements forestiers composés de pins laricio, hêtres et sapins, à végétation vasculaire dont l'association relève du $P o o-$ Fagetum-abietietosum, 1.7.2013, Pioli, A. (Hb. privé 20130701001).

Espèce peu fréquente en Corse, connue des régions de Bavella, Piedicroce et vallée du Manganello (Sotiaux et al. 2007).

Isopterygiopsis pulchella (Hedw.) Z. Iwats.

- 2A, Forêt territoriale de Marmanu, en bordure du chemin de randonnée GR20, tourbière de pente, avec une configuration de pozzines, à cortège floristique de plantes vasculaires se rattachant au PinguiculoTrichophoretum cespitosi narthecietosum, boisée par bouquets, sapins, pins laricio, hêtres, avec une présence importante des aulnes odorants, à couvert de 4/10 [NM132528], 1457 m, 3.10.2013, Pioli, A. s.n. (Hb. CBNC).

Espèce rare, signalée seulement à l'Ospedale (Sotiaux et al., 2007).

Kiaeria starkei (F. Weber \& D. Mohr) I. Hagen

- 2A, Forêt territoriale de Marmanu, en bordure du chemin de randonnée GR20, cortège floristique vasculaire ayant des affinités avec le groupement de marais à laiches Caricetum intricatae caricetosum, mais aussi avec la mégaphorbiaie du Valeriano rotundifoliae-Adenostyletum briquetii [NM133528], $1390 \mathrm{~m}$, 3.10.2013, Pioli, A. s.n. (Hb. CBNC). 
Espèce rare, signalée dans l'île sans précision de localité (Sotiaux et al., 2007).

\section{Philonotis rigida Brid.}

- 2B, Forêt communale de Noceta [NM833703], bord de fossé humide, en touffe, dans un peuplement de pin laricio de Corse, à végétation vasculaire dont l'association relève du Galio-Pinetum laricii luzuletosum, 24.6.2012, Pioli, A. (Hb. privé 20120624001).

Espèce peu fréquente en Corse, citée pour la région d'Ajaccio à Calcatoggio (CAmus, 1902; Sotiaux et al., 2007).

Plagiothecium platyphyllum Mönk.

- 2B, Forêt de Valdoniellu, abords du lac de Lavigliolu, petite falaise sur la droite du lac (face au déversoir) [NM964795], $1834 \mathrm{~m}$, grosse touffe sur paroi rocheuse, avec suintements importants, zone rocheuse d'altitude aux alentours du lac dans les taillis de l'aulnaie odorante, 29.7.2011, Pioli, A. (Hb. privé 20110729001).

Espèce peu fréquente en Corse, connue sur le massif du Renoso et le plateau du Coscione (HÉBrard, 1986).

Poblia cruda (Hedw.) Lindb.

- 2A, Forêt territoriale de Sant'Antone, tourbière de pente à végétation vasculaire dont l'association relève du Caricetum intricatae caricetosum, située dans un peuplement forestier de pin laricio et sapin dominant avec un sous étage de hêtre [NM157529], $1270 \mathrm{~m}$, 11.9.2013, Pioli, A. s.n. (Hb. CBNC).

Espèce rare, signalée au-dessus de Bastia, au col de Vizzavona, au Monte d'Oro, dans la vallée de la Restonica, à Bonifato et dans la forêt de Valdoniello (CAmus, 1895, 1903; SARrasat, 1931).

\section{Polytrichum commune Hedw.}

- 2A, Forêt territoriale de Sant'Antone, tourbière de pente à végétation vasculaire dont l'association relève du Caricetum intricatae caricetosum, située dans un peuplement forestier de pin laricio et sapin dominant avec un sous étage de hêtre [NM157529], $1270 \mathrm{~m}$, 11.9.2013, Pioli, A. s.n. (Hb. CBNC).

Espèce peu fréquente, signalée notamment au lac de Melo, au lac d'Oriente, aux lacs de Rina, dans le vallon de Tula, et au lac de Nino (Camus, 1902; Hébrard, 1986).

\section{Polytrichum strictum Brid.}

- 2A, tourbière aux alentours du col de Verde [NM150527], forêt territoriale de Sant'Antone, 1330 m, avec Sphagnum subnitens, Sphagnum palustre, Leucobryum glaucum, Scapania undulata, Scapania paludosa, milieu forestier à peuplement composé du pin laricio dominant, avec le sapin et le hêtre, les autres plantes vasculaires représentées par Erica scoparia, Betula pendula, accompagnées par de nombreuses cypéracées et joncacées, et présence de plusieurs pieds d'Huperzia selago, 29.4.2013, Pioli, A. (Hb. privé 2013042920); Forêt territoriale de Sant'Antone, tourbière de pente à végétation vasculaire dont l'association relève du Caricetum intricatae caricetosum, située dans un peuplement forestier de pin laricio et sapin dominant avec un sous étage de hêtre [NM157529], 1270 m, 11.9.2013, Pioli, A. s.n. (Hb. CBNC).

Espèce rare en Corse, citée au col de Vizzavona (CAmus, 1903; Sotiaux et al., 2007).

\section{Thamnobryum alopecurum var. gracillimum (Bot.) Dull}

- 2A, Forêt territoriale de Marmanu, en bordure du chemin de randonnée GR20, tourbière de pente, avec une configuration de pozzines, à cortège floristique de plantes vasculaires se rattachant au PinguiculoTrichophoretum cespitosi narthecietosum, boisée par bouquets, sapins, pins laricio, hêtres, avec une présence importante des aulnes odorants, à couvert de 4/10 [NM132528], 1457 m, 3.10.2013, Pioli, A. s.n. (Hb. privé $\mathrm{CBNC}$ ).

Variété nouvelle pour la Corse. Citée dans la flore italienne de CoRTina PEDRotTi (2005) comme assez rare en Italie.

Thuidium tamariscinum (Hedw.) Schimp.

- 2A, Forêt territoriale de Sant'Antone, tourbière de pente à végétation vasculaire dont l'association relève du Caricetum intricatae caricetosum, située dans un peuplement forestier de pin laricio et sapin dominant avec un sous étage de hêtre [NM157529], $1270 \mathrm{~m}$, 11.9.2013, Pioli, A. s.n. (Hb. CBNC).

Espèce rare, signalée à Bastia et Bonifato (CAmus, 1895).

\section{Conclusion}

Avec ces nouvelles découvertes, la bryoflore de l'île s'est accrue de trois nouvelles espèces (Barbilophozia attenuata, Jamesoniella autumnalis, Hygroamblystegium tenax) ainsi que d'une nouvelle variété (Thamnobryum alopecurum var. gracillimum (Bot.) Dull) et se compose donc dorénavant de 578 espèces comprenant 3 anthocérotes, 156 hépatiques et 419 mousses. De plus, de nouvelles stations d'espèces rares ou peu fréquentes permettent de compléter les données et de mieux appréhender leur répartition dans l'île. 


\section{Références}

Bischler, H. \& S. Jovet-Ast (1973). Les hépatiques de Corse, énumération, notes écologiques et biogéographiques. Rev. Bryol. Lichénol. 39: 43-153.

Camus, F. (1895). Notes sur les récoltes de M. P. Mabille en Corse. Rev. Bryol. 22/5: 63-74.

Camus, F. (1902). Note préliminaire sur un voyage bryologique en Corse. Rev. Bryol. 29/2: 17-26.

Camus, F. (1903). Muscinées recueillies en Corse en mai et juin 1901. Bull. Soc. Bot. France 48: 151-174.

Cortina Pedrotti, C. (2005). Flora dei muschi d'Italia, Bryopsida (II parte). Ed. Antonio Delfino.

DüLL, R. (1983). Distribution of the European and Macaronesian mosses (Hepaticophytina). Bryol. Beitr. 2: 1-114.

ECCB (1995). Red Data Book of European Bryophytes. European Committee for Conservation of Bryophytes (ECCB), Trondheim.

Gamisans, J. (1999). La végétation de la Corse. Edisud.

Geissler, P. (1981). Some aspects of high altitude bryoflora of Corsica. In: Szweyкошsкi, J. (ed.), New perspectives in bryotaxonomy and bryogeography: 119-122. Adam Mickiewicz University, Poznan.

Grolle, R. \& D. G. Long (2000). Annotated checklist of the Hepaticae and Anthocerotae of Europe and Macaronesia. J. Bryol. 22: 103-140.

Hebrard, J.-P. (1978). Contribution à l'étude de la végétation muscicale des rochers siliceux secs en haute montagne corse. Bull. Soc. Sci. Hist. Nat. Corse 627-628: 69-91.

Hebrard, J.-P. (1986). Note de bryologie corse: muscinées rares, méconnues ou nouvelles pour l'île. Bull. Soc. Bot. Centre-Ouest, n.s. 17: 151-167.

Hebrard, J.-P. (2005). New localities and ecology of Buxbaumia viridis (Buxbaumiaceae, Musci) in Corsica. Nova Hedwigia 80: 241-246.

Hill, M. O., N. Bell, M. A. Bruggeman-Nannenga, M. Bruges, M. J. Cano, J. Enroth, K. I. Flatberg, J. P. Framh, M. T. Gallego, R. Garilleti, J. Guerra, L. Hedenas, D. T. Holyoak, J. Hyvonen, M. S. Ignatov, F. Lara, V. Mazimpaka, J. Munoz \& L. Soderstrom (2006). An annotated checklist of the mosses of Europe and Macaronesia. J. Bryol. 28: 198-267.

Parriat, H. (1951). Les espèces alpines et boréales-alpines de la flore corse. Rev. Bryol. Lichénol. 20: 1-2.

Pioli, A. (2006). Contribution à l'inventaire de Buxbaumia viridis. Nouveaux habitats et nouvelles répartition en Corse. Bull. Soc. Sci. Hist. Nat. Corse 710/711: 43-58.
Ross, R. M., V. Mazimpaka, U. Abou-Salama, M. Aleffi, T. L. Blockeel, M. Brugues. M. J. Cano, R. M. Cros, M. G. Dia, G. M. Dirkse, W. El-SaAdawi, A. Erdag, A. Ganeva, J. M. GonZalez-Mancebo, I. Herrnstadt, K. Khalil, H. Kurschner, E. Lanfranco, A. Losada-Lima, M. S. Refai, S. RodriguezNunez, M. Sabovljevic, C. Sergio, H. M. Shabbara, M. SimSim \& L. Solderstrom (2007). Hepatics and Anthocerotes of the Mediterranean, an annoted checklist. Cryptog. Bryol. Lichénol. 28: 351-437.

Ross, R. M., V. Mazimpaka, U. Abou-Salama, M. Aleffi, T. L. Blockeel, M. Brugues. R. M. Cros, M. G. Dia, G. M. Dirkse, I. Draper, W. El-SaAdawi, A. Erdag, A. Ganeva, R. Gabriel, J. M. Gonzalez-Mancebo, C. Granger, I. Herrnstadt, V. Hugonnot, K. Khalil, H. Kurschner, A. Losada-Lima, L. Luis, S. Mifsud, M. Privitera, M. Puglisi, M. Sabovljevic, C. Sergio, H. M. Shabbara, M. Sim-Sim, A. Sotiaux, R. Tacchi, A. Vanderpoorten \& O. Werner (2013). Mosses of the Mediterranean, an annoted checklist. Cryptog. Bryol. Lichénol. 34: 99-283.

Sarrasat, C. (1931). Muscinées récoltées en Corse au cours de la session de la Société botanique de France du 4 au 14 août 1930. Bull. Soc. Bot. France 78: 689-692.

Schumacker, R. \& J. VANA (2005). Identification keys to the liverworts and hornworts of Europe and Macaronesia. Sorus, Poznan.

Skrzypczak, R. (2005). Contribution à l'étude de la bryoflore du Cap Corse. Bull. Soc. Bot. Centre-Ouest ser. 2, 35: 581-582.

Sotiaux, A., J. Enroth, S. Olsson, D. Quandt \& A. VanderPOORTEN (2009). When morphology and molecules tell us different stories: a case-inpoint with Leptodon corsicus, a new and unique endemic moss species from Corsica.J. Bryol. 31: 186-196.

Sotiaux, A., A. Pioli, A. Royaud, R. Schumacker \& A. VAnderpoorten (2007). A check list of the bryophytes of Corsica (France): new records and a review of the literature. J. Bryol. 29: 41-53.

Sotiaux, A., O. Sotiaux \& A. Vanderpoorten (2008). Additions to the bryophyte flora of Corsica. Cryptog. Bryol. Lichénol. 29: 267-274. 